\title{
Design and Implementation of computer Aided Decorative Design System
}

\author{
Cao Zhang, Ling Chen \\ \{zhangcaowh@163.com\}
}

College of art and design wuchang shouyi university, wuhan 430064, China

\begin{abstract}
This paper fully considers the system in the technology of inheritance and innovation, to fully grasp the principles and concepts of the decorative arts, as well as the basic process of computer aided design based on, the system of research and analysis of demand. Then, according to the system requirements analysis and feasibility study, and the overall design of the system, including the system function division and module design, on this basis, the use of Mel scripting language system. At last, the system is tested and further improved. This topic comes from the actual demand, combined with the actual application requirements, the paper mainly introduces the design and implementation process of the computer aided design system based on the prototype method and MEL language. The system is based on the knowledge of software engineering as the guiding ideology of the design and development of the system, the main function of the system includes user management system, modeling module works, the material setting module, lighting layout module, script sharing and management module and so on. In addition, the paper uses the UML modeling technology to analyze the system, to ensure the system design of the normative. Papers in the system architecture design, module realization and system integration, basically follow the rational function, the humanized principle, extension principle and the principle of modular design. This ensures that the system maintenance and reliability; the of decorative art of material, scale, form and environment characteristics and computer aided system were combined effectively, ensure the availability of system functions and good user experience.
\end{abstract}

Keywords: Auxiliary decoration; Mel; J2EE; system test

\section{Introduction}

At present, the computer aided design and decoration system has become people in an important platform for the family decoration and art design, people can be in accordance with your favorite colors and shapes and hobbies to decorative design, improve the design efficiency, and compared with the traditional hand drawn and computer aided design will be more simple and accurate. The following first introduces the background and significance of this topic, the system to achieve the goal and so on [1]. Decoration design is an essential part of people's house decoration, but also to show people's pursuit of beauty and taste, it is to meet the people's spiritual, material life needs of the arts. Especially in recent years, decoration design for artistic quality have higher requirements, design content involves requirements has 
a high technical content, if still use the traditional manual drawing method, not only the efficiency is high, the emergence of new technology, new material and, and puts forward the new requirements, hand drawing has been difficult to meet people's needs. Therefore, it is needed to design a computer aided design system to replace the traditional manual drawing method, which is convenient for designers to improve the design efficiency. With the people in the decoration industry requirements continue to increase, and decoration industry in the people's life scenarios more and more rich, taking technology of the computer and the decoration technique for supporting the decoration system also experience, from the low level to the mature stage. In the use of computers as a tool to assist the design and decoration of early, is 20 century the sixties and seventies, due to the limitation of the development level of computer software and hardware at that time, and user cannot truly understand the principle of computer, lead renderings of the completion of the initial period in the picture of the sense of reality and the production efficiency are unable to hand-painted renderings and compared. Therefore, this form of expression was not known as the mainstream of this period. In the 90s of the 20th century, computer software and hardware have rapid development, computer in the decoration design in the proportion of participation is gradually increasing, but the period of design production staff are from the computer professional, only very few received professional training in aesthetics, to result more performance out of the real, and not as a kind of true art. At the beginning of the 21 st century, a lot of decoration designers and the educators began to gradually realize mastery of CAD is decoration design important content is new period to the interior designers a new requirements, and at the same time, the design professional colleges and universities began to set up systematic computer software, curriculum design, the era of computer aided drawing, both in quality or production efficiency has been greatly improved, computer aided software industry into the decoration design expression of a subject [2].

\section{Introduction of System Related Technologies}

Under normal circumstances, the decorative art mainly includes 4 kinds of characteristics, including materials, scale, form and environment. Decorative art is generally based on the material performance, materials play a decisive role in the art of decoration, so designers must first before the creation of design to determine the material selection. Art Deco materials about artificial composite materials and natural materials, decoration designers understanding of the material and the ability to control the size of, in the process of artistic creation plays a very important role. Therefore, designers need to be familiar with the characteristics of the material, including the strong toughness, corrosion, shrinkage, etc., so that the need for a better choice of material. Also material has the color, shape and other external in characteristics, abstract impression in the minds of the designers, to the designer's creative thinking also has great influence. The most outstanding decorative artist usually after long time consideration, testing and practice, through a wide variety of materials and information collection, the depth of the familiar features, to think about these materials to where and how to show the most beautiful, the scale is also very important for the Art Deco of and its impact on the final visual aesthetic effect is very big. The scale is largely determined by the environment. Such as decorative murals, the main difference between it and the case on the painting is decorated with murals of scale greatly, so it is often to appreciate those who generate strong impact force and the impact force directly stimulate appreciation of the optic 
nerve, to convey the appreciation of the cerebral cortex, then in the mind left very deep feelings and impressions. Through this process, the realization of the decoration of the United states [3].

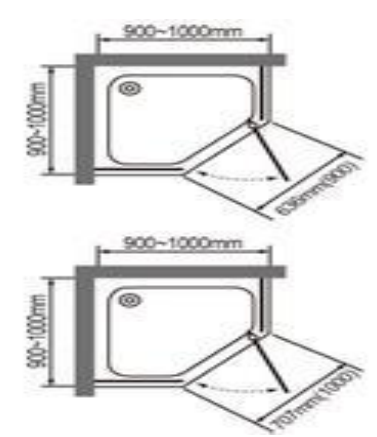

Fig. 1. Computer aided design sketch map

\subsection{Computer Aided Design}

In the decorative design in this process, designers are generally required to draw a lot of renderings to the entrusting party, construction personnel that the intention of the designers and the idea of creation, the early decoration design uses is with paper, pen, hand, rulers as tools of artificial design, this kind of artificial drawing not only delay the decoration design cycle, but also hindered the designers to give full play to. With the rapid development of the computer, some computer graphics design software is playing an invaluable role, they do a good assistant for decorative design. In recent years, a variety of computer graphics software systems have been born. At present, the computer has begun to affect to the traditional design methods, people began with the aid of the computer to accomplish a lot of mechanical hand times, to spend a lot of manpower and material resources to achieve the design work, the use of computer aided design is an important characteristic of the modern decoration design. In the decoration design, the computer is playing an important role. Decorative design using computer aided design, there will be a lot of benefits. Among them, the most meaningful is to the decoration designers provide very large free play space, designers can by computer designers can grasp the picture material via a computer any spell stickers, combination, simple, convenient various attempts, in order to make the current architecture environment and the matching of a. This is a convenient way to try and help to give inspiration to the creator. Such as Kaiser Sargent "why peace has not yet achieved" in, combination of head with the dove of peace, with relative and the difference between the two parties mutual collocation, creating and building the most matching decoration, also very strong show the theme of peace to the needs of the society. 


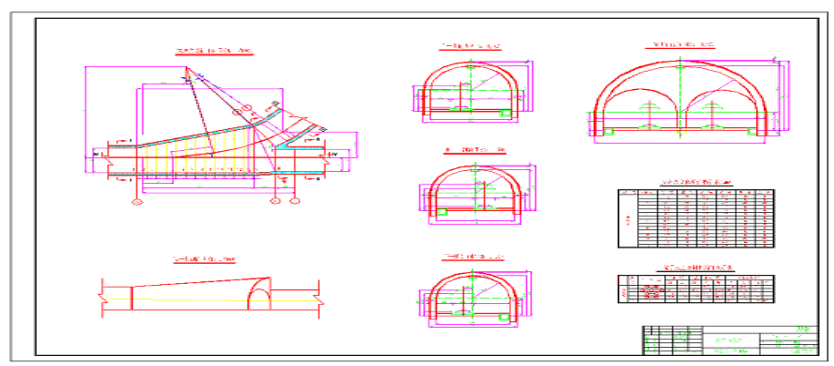

Fig. 2. Computer aided design of CAD drawing

\subsection{Introduction and Analysis of J2EE Technology}

The technology of J2EE and database technology to design and develop Mel scripts sharing module, for different developers can share the script file, in order to realize the online viewing, download and upload script file. Only in this way can improve the working efficiency of the entire team, reduce the cost of development. In java servlet is executed on the server side code, has a very good system portability, regardless of the selection of the windows operating system and the Linux operating system, application developers can development servlet program will be deployed to the executed in the operating system, and the security code is very good. These are also a lot of people uses Java to develop the system is one of the important reasons. At the same time, servlet with powerful function, based on java servlet can fully reflect the technical advantages of Java API, sometimes developers some query software, can use JNDI API to develop. And Servlet can use JDBC to connect to the database, distributed development. Another advantage of servlet is its performance was satisfactory, when the servlet executed once, save for a period of time in the cache, when the same request, the servlet will use different thread to handle, in performance would have improved dramatically, and the server will automatically remove the long residence time and no implementation of the servlet. In addition, Servlet also has the characteristics of type checking, and can use the garbage collection of Java, make Servlet avoid the problem of memory management, reduce the burden on the development of the programmer [4].

\section{System Design and Implementation}

The purpose of software project management is to make use of resources effectively, to ensure that the software is delivered on schedule. The software design is based on the requirements of the software, design software system structure and data structure, determine the composition of the program module and the relationship between the modules. In this paper, mainly using the top-down design method to design the system, first of all, on the system to solve the problem of "top-level" description, which is then decomposed into different parts to further find detail information to solve the problem. On this basis, repeat the above steps to break down the steps into smaller steps. Using this design method, the overall design of the system can be gradually refined into each line of the script. This system is designed and developed according to the customer's requirements, and has certain pertinence and particularity. At present, there is no readily available software products to meet the demand, and the prototype method is used to develop the project. The work of specific 
development projects in the overall design, detailed design can be divided into interface design and making, function development, performance tuning, based on user feedback adjustment function and ease of use four stage, after the end of the first three phases of the work should be submitted separately prototype interface, function prototype, through the performance test of the function prototype. Through three representative stages of the prototype system, customers can well grasp the progress of the project, and to ensure that the development of the system to meet the business needs and performance requirements. This system is a computer aided decoration design system based on Mel, in order to improve the system in the future the maintainability and scalability, you first need to the system function module division, which also has conducive to the development of modular system, but also can improve the code reusability. In the script sharing module needs to provide users with online download and upload file function, which requires the use of database technology to achieve the storage and sharing of information, which means that the data modeling. In the field of software engineering and software management, the concept of database design, database design is on the system in the future use of the environment and facing the business needs to establish that can carry a data storage and query a database environment. Under normal circumstances, the design process of the database mainly includes five stages, respectively is: demand analysis, the outline design, logic structure design and analysis of the physical model and data to create, in the application database is mainly for storing and querying the data provided by the underlying service, but when the relatively large amount of data requires further consideration of the database can bear the pressure and its performance. In an information management system, if the underlying database failure, the entire system can not run, the database in this system mainly includes the user and the script part of the data information [5].

\section{Conclusion}

In the field of decoration design, in order to improve the quality of the designer's work, shorten the design cycle, speed up the design efficiency, can use the current relatively advanced computer technology as an auxiliary means of design. Computer technology and its application and traditional Chinese decorative design combined to create a unique, and in accordance with the current of contemporary aesthetic needs for daily needs, decorative design products, is worthy of study and have important practical significance is a major task. The purpose of this paper is to use simple operations to assist designers to finish the design of the decoration works better, through the computer to decorative shapes attempt to obtain better design ideas. Furthermore, the decoration designers can take some frequently used design module or for goods stored, as reusable creation material, cost savings for the early, can provide good technical reference, provides a more convenient operation and also to the creation of decorative works.

\section{References}

[1] Peng Xia, Zhu Ping, Ren Yongchang. Comparative analysis of software design tools in the study of. Computer technology and development. No. 03. (2013) 
[2] Lu Xinxin, Lu Jianjun, Zhao Anxin. Design of. Coal technology self-service system of intelligent terminal based on coal mine. No. 01. (2013)

[3] Gong Xueqing, Jin Cheqing, Wang Xiaoling, Zhang Rong, Zhou Aoying. Data intensive science and Engineering: requirements and challenges. Journal of computer science. No. 08. (2012)

[4] Wang Rui, Guo Jingshu, ye Rong. Furnishings art presentation and role in the interior design. Kunming Metallurgy College School Journal. No. 03. (2012)

[5] Years of pure, Feng Zhaoyang, Xi Hongming, Liu Huanmin, Wang Hua. Research and implementation of real time visualization system for space launch based on. STK Journal of system simulation. No. 04. (2012) 